\title{
Análise de ferramentas de suporte tecnológico para simulação da dinâmica da cobertura e uso do solo
}

\author{
Jean Samarone Almeida Ferreira ${ }^{1}$, Ana Paula Lüdtke Ferreira ${ }^{1}$ \\ ${ }^{1}$ Programa de Pós-Graduação em Computação Aplicada \\ Universidade Federal do Pampa \\ Avenida Maria Anunciação Gomes de Godoy nº 1650 - Bagé - RS - Brazil \\ \{jeanferreira, anaferreira\}@unipampa.edu.br,
}

\begin{abstract}
Three softwares for land use and cover change (LUCC) are analyzed in this work, within the scope of the Rio Grande do Sul's Campanha region landscape evolution. Data acquisition and classification processes are shown and the main advantagens and drawbacks of each tool, concerning simulation, are emphasised. Our purpose is to help researchers as well as suggest sofware development trends and needs to fill this particularly gap.
\end{abstract}

Resumo. Este trabalho analisa três ferramentas de suporte computacional para a simulação da dinâmica da cobertura e uso do solo, dentro de um projeto para análise de evolução da paisagem na região da Campanha do Estado do Rio Grande do Sul. O processo de aquisição e classificação de dados prévio é apresentado e as vantagens e lacunas nas ferramentas livres e gratuitas analisadas são evidenciadas. Pretende-se com a análise realizada contribuir com os pesquisadores dessa área e apontar necessidades existentes para o devido suporte tecnológico.

\section{Introdução}

A cobertura do solo diz respeito aos elementos presentes na superfície física da Terra, constituídos a partir de fenômenos naturais ou antrópicos. O conjunto de elementos que fazem parte da cobertura do solo inclui vegetação natural e cultivada bem como infraestruturas criadas pelo homem. O uso do solo é definido como a transformação das paisagens naturais para utilização humana ou das alterações desse uso em áreas com ocupação humana [Camacho and Pérez-Barahona 2015] e inclui tanto a forma como as razões pelas quais os atributos biofísicos da Terra são modificados. O uso do solo influi diretamente na sua cobertura, tanto por alterações intencionais (construção de áreas urbanas, infraestrutura viária, atividades agropecuárias) como não intencionais decorrentes das estratégias de ocupação escolhidas (degradação do solo, poluição ambiental, aspectos sociais, políticos e econômicos). A dinâmica do uso do solo indica a mudança na cobertura e uso ao longo do tempo [Gomarasca 2009], provocada pela ação antrópica. O estudo da dinâmica do uso do solo fornece informações sobre o passado e permite inferências sobre o futuro, permitindo que políticas de uso possam ser formuladas, evitando atempadamente alterações não desejadas (poluição, degradação do solo, ocupação urbana em áreas de risco, por exemplo). A combinação de indicadores de uso do solo com indicadores sociais e econômicos pode apontar caminhos interessantes para o desenvolvimento de políticas públicas (geração de empregos e incentivos para áreas específicas) maximizando indicadores sociais e econômicos da região de estudo. Aspectos ambientais globais 
também são influenciados por dinâmicas locais do uso do solo: poluição e propagação de doenças, por exemplo, não são restritas aos seus locais de origem [Foley et al. 2005].

O estudo da dinâmica do uso e cobertura do solo exige a modelagem espacial da superfície da região de estudo atrelada a dados temporais sobre a cobertura, com outros indicadores (econômicos, sociais, ambientais, etc.) para inferência de informações para estabelecimento de políticas para uso de áreas urbanas e rurais. Dados de cobertura do solo são obtidos por sensoriamento remoto ou coleta de dados locais, com posterior aplicação de técnicas de reconhecimento de imagens, inferência e análise temporal. A literatura apresenta técnicas clássicas de análise com aplicação neste contexto, entre as quais cadeias de Markov, redes neurais, sistemas fuzzy, redes bayesianas, bem como combinações dessas e de outras técnicas. O uso de sistemas de informações geográficas (SIG), que suportam a construção de mapas e modelos de informação georreferenciada e manipulação imagens, é indicado devido à característica de espacialidade dos sistemas ambientais [Christofoletti 1999], permitindo a análise de fenômenos, variáveis ou eventos com referência espacial conhecida [Burrough 1986]. As ferramentas de suporte para aquisição, tratamento e classificação de dados ainda não são suficientes para projetos envolvendo análises temporais. A simulação desses processos é necessária, visto não ser possível operar diretamente sobre a realidade. Simulações objetivam estudar as evoluções futuras de um sistema, dentro das condições de variabilidade dos processos envolvidos, permitindo conclusões consistentes do ponto de vista matemático [Law 2007, Bellomo 2008]. Simulações ainda facilitam o entendimento dos processos simulados pela sua visualização, facilitando a comunicação entre pesquisadores e públicoalvo dos resultados de pesquisa [Boulic and Renault 1991].

A escolha das ferramentas computacionais impacta o tipo e a qualidade das conclusões obtidas e a facilidade de execução do trabalho. Sistemas de software possuem diversos níveis e características de desenvolvimento, tornando alguns mais adequados para aplicações específicas. Usualmente, grupos de pesquisa já trabalham com SIG para registro e manipulação de informações georreferenciadas. Projetos que demandam ferramentas para a análise de dinâmica da evolução do uso do solo precisam de análise de custo-benefício, a partir dos requisitos do projeto.

A unidade de estudo é a bacia hidrográfica do Arroio Piraí, que é uma sub-bacia do Rio Negro, a qual abrange os municípios de Aceguá, Bagé, Candiota, Dom Pedrito e Hulha Negra, no estado do Rio Grande do Sul. Os principais usos da água são irrigação, abastecimento humano e dessedentação animal, conforme a Secretaria do Ambiente e Desenvolvimento Sustentável [SEMA 2018], indicando que o uso do solo está fortemente atrelado à atividade agropecuária. Foram coletadas e analisadas imagens da região em dois anos consecutivos.

O objetivo geral deste trabalho é comparar três ferramentas de suporte tecnológico à simulação da mudança no uso e na cobertura do solo. Para a comparação é feito um estudo nas mudanças ocorridas entre os anos de 2016 e 2017 na bacia hidrográfica do Arroio Piraí, região da Campanha do Rio Grande do Sul. Para a simulação foram classificados quatro tipos de uso ou cobertura (água, floresta, campo e agricultura) para os anos de 2016 e 2017, usando três variáveis espaciais (elevação, declividade e distância da água), a partir do SRTM (Shuttle Radar Topography Mission) [Farr et al. 2007]. A execução da simulação foi feita usando três ferramentas diferentes de simulação: MOLUSCE 
[MOLUSCE 2018], TerraME [TerraME 2018] SIMLANDER [SIMLANDER 2018]. A contribuição deste trabalho inclui a análise das ferramentas utilizadas, do ponto de vista da usabilidade e técnicas de análise implementadas, como forma de contribuir para projetos na mesma área e facilitar o trabalho dos pesquisadores que vierem a se engajar nessa área de pesquisa. O restante deste texto está organizado como se segue: a Seção 2 apresenta a metodologia e as técnicas de análise utilizadas no desenvolvimento as simulações e a Seção 3 apresenta as considerações finais e os trabalhos futuros.

\section{Materiais e Métodos}

\subsection{Aquisição e classificação de dados}

O trabalho seguiu uma metodologia aplicada e exploratória, buscando entender as interfaces de instalação e funcionamento dos programas utilizados. Os processos de aquisição, classificação e armazenamentos de imagens foram efetivados no software QGIS [QGIS 2018], projeto oficial da Open Source Geospatial Foundation [OSGEO 2018], software multiplataforma, que suporta diferentes formatos de dados vetoriais, raster e bases de dados. A seleção das imagens foi feita no Copernicus Open Access Hub [COPERNICUS 2018], que é o programa de observação da Terra liderado pela Comissão Europeia [EEA 2018]. Copernicus é um conjunto de sistemas de aquisição de dados, combinando sensoriamento remoto e observações locais (estações em terra, sensores aéreos e marítimos) com propósito de oferecer dados confiáveis, atualizados e de acesso aberto em seis áreas temáticas: terra, água, atmosfera, mudanças climáticas, gestão de emergência e segurança. No cerne do programa está uma série de seis novos satélites (Sentinels) [Sentinel 2018], em operação (Sentinel-1A, Sentinel-1B, Sentinel2A, Sentinel-2B, Sentinel-3A e 3B) e o restante com previsão de entrada em órbita até 2025. O Sentinel-2 é um satélite complementar ao Landsat e apresenta algumas vantagens, como a resolução espacial (10, 20 e 60 metros), faixa imageada $(290 \mathrm{Km})$ revisitada a cada 10 dias (um satélite) e 5 dias (dois satélites), além de 13 bandas espectrais, complementares aos dados adquiridos pelo Landsat 8 -OLI e Landsat 7 Enhanced Thematic Mapper Plus (ETM +). Essas características fazem do Sentinel-2 um produto interessante para análises de uso e cobertura da terra. As imagens utilizadas na classificação foram adquiridas em 12/11/2016 e 12/11/2017, pelo Sentinel-2A e Sentinel-2B, obtendo-se 13 bandas espectrais com resolução espacial de $10 \mathrm{~m}, 20 \mathrm{~m}$ e $60 \mathrm{~m}$, conforme ilustrado na Tabela 1. No processo de classificação supervisionada, foram utilizadas as bandas: 2, 3, $4,5,6,7,8,8 \mathrm{a}, 11$ e 12 .

O processo de classificação fez uso do plugin de código aberto SCP (semiautomatic classification plugin) [Congedo 2018], que permite a classificação supervisionada de imagens de sensoriamento remoto. O processo de classificação compreende as fases de importação das imagens, correção atmosférica, delimitação de áreas de treinamento, definição das assinaturas espectrais, classificação e verificação da acurácia das classificações. As imagens de alta definição (formato jpeg 2000 ou jp2), após importação para o QGIS, foram corrigidas dos efeitos da atmosfera no recorte retangular da área que compreende a bacia hidrográfica do Arroio Piraí. Três composições [NASA 2018] $\mathrm{RGB}=432, \mathrm{RGB}=742$ e $\mathrm{RGB}=321$ foram usadas para auxílio na análise visual e delimitação da base de treinamento (Figuras 1(a), 1(b) e 1(c)).

O objetivo da classificação é produzir um mapa temático da cobertura do solo. A demarcação de áreas de treinamento para cada classe de cobertura de terra identificada na 
Tabela 1. Bandas do Sentinel 2

\begin{tabular}{lll}
\hline Bandas Sentinel-2 & Comprimento de Onda Central $[\mu \mathrm{m}]$ & Resolução [m] \\
\hline Band 1 - Coastal aerosol & 0.443 & 60 \\
\hline Band 2 - Blue & 0.490 & 10 \\
\hline Band 3 - Green & 0.560 & 10 \\
\hline Band 4 - Red & 0.665 & 10 \\
\hline Band 5 - Vegetation Red Edge & 0.705 & 20 \\
\hline Band 6 - Vegetation Red Edge & 0.740 & 20 \\
\hline Band 7 - Vegetation Red Edge & 0.783 & 20 \\
\hline Band 8 - NIR & 0.842 & 10 \\
\hline Band 8A - Vegetation Red Edge & 0.865 & 20 \\
\hline Band 9 - Water vapour & 0.945 & 60 \\
\hline Band 10 - SWIR - Cirrus & 1.375 & 60 \\
\hline Band 11 - SWIR & 1.610 & 20 \\
\hline Band 12 - SWIR & 2.190 & 20
\end{tabular}

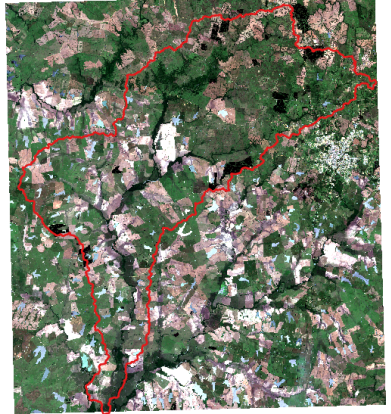

(a) $\mathrm{RGB}=432$

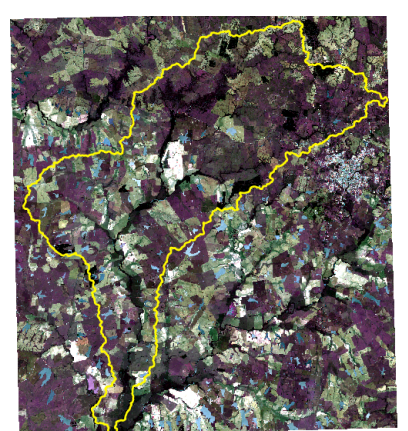

(b) $\mathrm{RGB}=543$

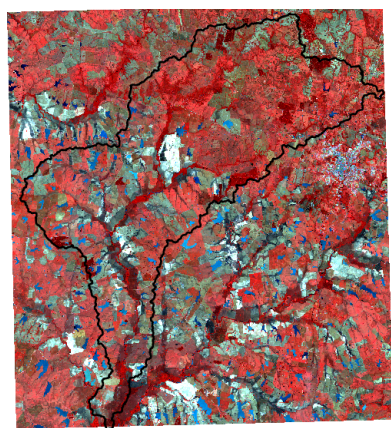

(c) $\mathrm{RGB}=853$

Figura 1. Composições RGB

imagem foi efetuada por polígonos desenhados em áreas homogêneas, nas quais todos os pixels pertencem à mesma classe de cobertura. O algoritmo region growing, disponível no SCP, foi usado para maior rapidez e acurácia, selecionando pixels a partir de uma semente, considerando a similaridade (ou distância) espectral. A classificação compara as características espectrais de cada pixel com as características espectrais das classes de treinamento (algoritmo de distância mínima, com função discriminante adaptada de [Richards and Jia 2006]). As assinaturas espectrais geradas para cada um dos alvos podem ser vistas na Figura 2, sendo no eixo $y$ os valores de reflectância e no eixo $x$ o comprimento de onda. As linhas verticais são as 10 bandas do Sentinel-2. O conjunto de treinamento obtido costa de 40 polígonos de tamanhos variados, correspondendo a cerca de $5 \%$ da área total. A verificação da acurácia foi realizada frente a novas amostras para cada um dos alvos.

\subsection{Softwares para Simulação da Dinâmica da Ocupação e Uso do Solo}

Para execução deste trabalho foram avaliados três softwares de simulação que permitiam a interação com os dados produzidos do QGIS, a saber: TerraME, SIMLANDER e MOLUSCE. A seguir as ferramentas listadas serão discutidas a respeito de suas potencialidades e/ou fragilidades.

A plataforma TerraME (TerraLib Modelling Environment) permite o desenvolvimento e suporte de modelagem ambiental espacial dinâmica, fazendo parte do framework 


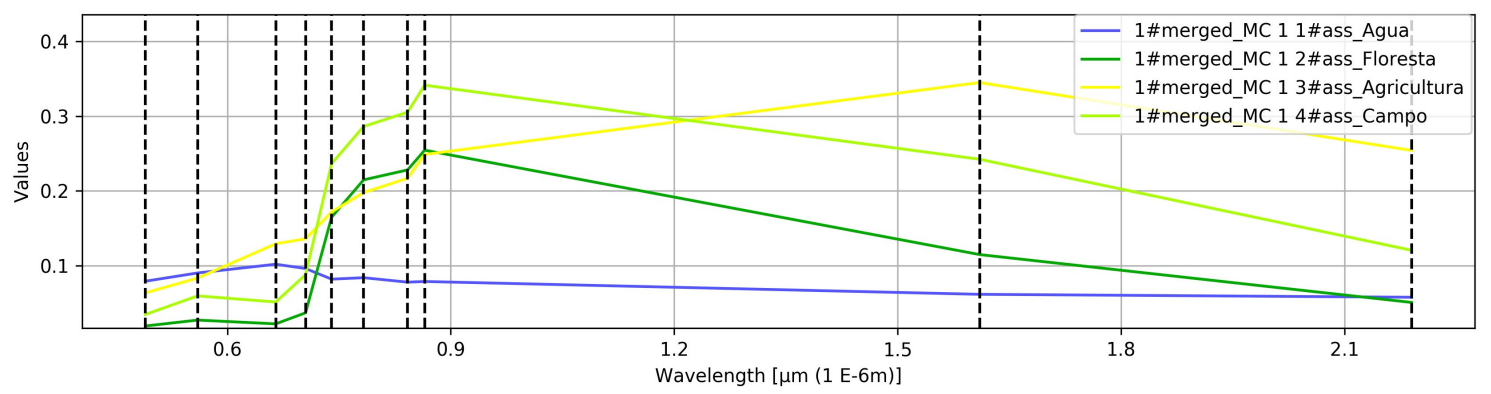

Figura 2. Assinaturas espectrais para água, floresta, agricultura e campo em $12 / 11 / 2016$

TerraLib [Câmara et al. 2001]. Suporta modelos de computação baseados em autômatos celulares e conceitos de autômatos celulares aninhados [de Senna Carneiro 2006]. O TerraME, associado ao SIG Terralib, permite a construção de modelos espaciais, resultando em mapas de distribuição espacial de um padrão ou de uma variável contínua e a simulação em duas dimensões de espaços celulares (regulares e irregulares). Os modelos podem ser implementados tanto em $\mathrm{C}++$ como na linguagem de programação TerraME Modeling Language, extensão da linguagem LUA [Ierusalimschy et al. 1996]. O TerraMe apresenta vantagens conceituais: a) modelagem multi-escala (escalas aninhadas); b) modelo espacial, que permite a integração com base de dados geográficos (Terralib), c) modelo temporal, com um escalonador de eventos; e d) modelo comportamental, definido com agentes ou autômatos celulares. Apesar das vantagens conceituais em relação aos demais softwares estudados, neste trabalho ficou inviável sua utilização, pois não foi possível utilizar uma versão binária ou mesmo compilar uma versão, que executasse de forma adequada sobre a plataforma Linux.

SIMLANDER (SIMulation of LAND Use Change $\boldsymbol{E}$ using $\boldsymbol{R}$ ) permite o desenvolvimento de um modelo de uso da terra baseado em autômatos celulares para execução em $\mathrm{R}$ [R Core Team 2018], consistindo em um script para o ambiente de software R. Por ser um software em desenvolvimento, apresenta várias limitações como: a) é um protótipo, sem qualquer garantia de funcionamento; b) pouco tempo de desenvolvimento; e c) sem interface gráfica. Para testar o SIMLANDER com os dados de exemplo foi necessário instalar alguns pacotes do $\mathrm{R}$, fazer pequenas correções na sintaxe dos comandos $\mathrm{R}$ do script disponibilizado. O manual é muito simples, tratando somente da instalação.

MOLUSCE (Modules for Land Use Change Evaluation) é um plugin projetado para analisar, modelar e simular mudanças no uso da terra. O MOLUSCE incorpora algoritmos que podem ser usados em análise de uso e cobertura do solo - urbanas e florestais. O processo de utilização do MOLUSCE consiste nas fases de entrada de dados, avaliação de correlações, análise de variação de área, escolha dos métodos de modelagem, simulação e validação. O módulo de entrada de dados recebe mapas de cobertura e uso do solo de diferentes épocas e dados de variáveis socioeconômicas e ambientais, tais como o sistema viário, rede hidrográfica, topografia e populações. O módulo de avaliação de correlações dispõe de recurso para análises de correlação entre as diversas variáveis de entrada. O módulo de análise de variação de área calcula a variação no uso e ocupação do solo entre dois períodos distintos de tempo, produzindo matrizes de transição para o uso e ocupação e mapas de alterações do uso do solo. O módulo de modelagem possui 
quatro métodos para a modelagem de potencial de transição do uso e ocupação do solo: redes neurais artificiais, regressão logística, avaliação multi-critério e pesos de evidência. O módulo de simulação apresenta um mapa de potencial de transição, função certeza (em caráter experimental) e resultados da simulação. Um mapa de uso e cobertura do solo simulado é produzido com base em um modelo de autômato celular. O módulo de validação incorpora estatísticas kappa (kappa padrão, histograma kappa e locação kappa), as quais podem ser utilizadas para validar a acurácia dos mapas simulados de uso e cobertura do solo.

\subsection{Análise do Uso e Cobertura do Solo}

Para a análise do uso e cobertura da terra foi utilizado um modelo digital de elevação (MDE) com resolução (aproximadamente 1 arco/segundo) de 30 metros, tendo como origem os dados de radar da missão Shuttle Radar Topography Mission (SRTM). A variável distância do corpo d'água foi gerada utilizando o módulo r.grow do GRASS [Neteler and Mitasova 2008], a partir da malha hidrográfica extraída do MDE, calculando as distâncias entre os elementos da malha hidrográfica e os divisores de água. A variável declividade é gerada também a partir do MDE, utilizando o módulo r.slope.aspect do GRASS, e consiste na inclinação da superfície do terreno em relação à horizontal, ou seja, a relação entre a diferença de altura entre dois pontos e a distância horizontal entre esses pontos. As Figuras 3(a), 3(b) e 3(c) apresentam, respectivamente, os mapas das três variáveis espaciais utilizadas.

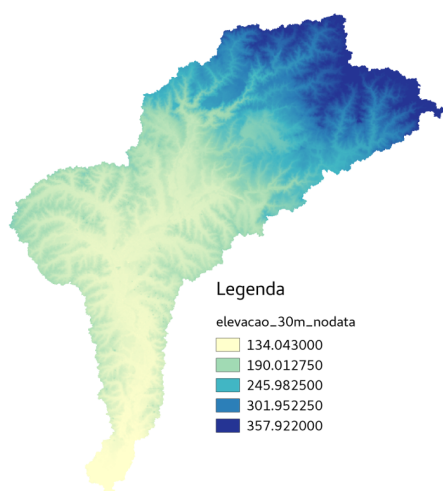

(a) Elevação (m)

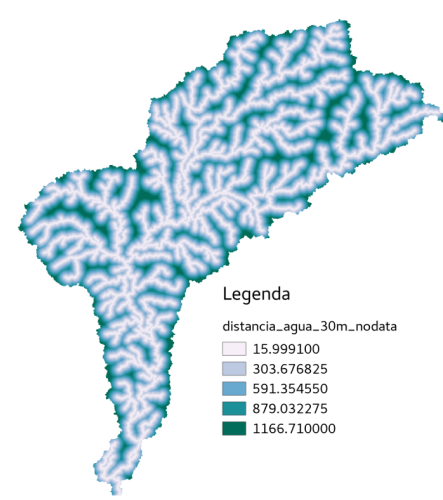

(b) Distância (m)

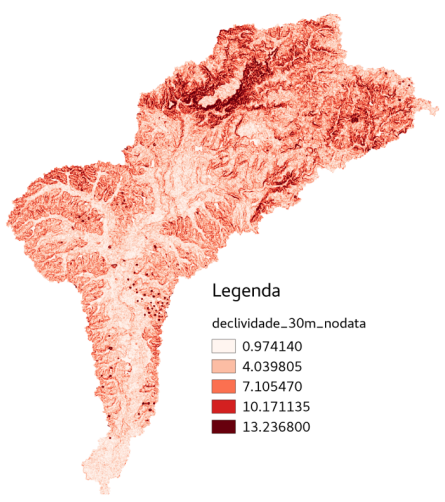

(c) Declividade $(\%)$

Figura 3. Variáveis Espaciais

Nas Figuras 4(a) e 4(b) são mostrados os resultados da classificação supervisionada, para os anos de 2016 (inicial) e 2017 (final), além das estatísticas de classe 4(c). A tabela de estatísticas de classes mostra as áreas inicial e final de cada uso/cobertura da terra alterado. Antes dos cálculos é feito um teste na geometria dos arquivos matriciais de entrada e também é importante que a área sem dados nos arquivos esteja identificada como NDV (no data value), para que não seja computada como classe de dados.

Após a entrada de dados, cálculo de estatísticas das classes, é possível fazer a análise de variação de área e calcular a matriz de transição, apresentada na Figura 5(a), que mostra a proporção de pixels modificados de um para outro uso/cobertura e permite a geração um mapa representando as mudanças, conforme Figura 5(b). 


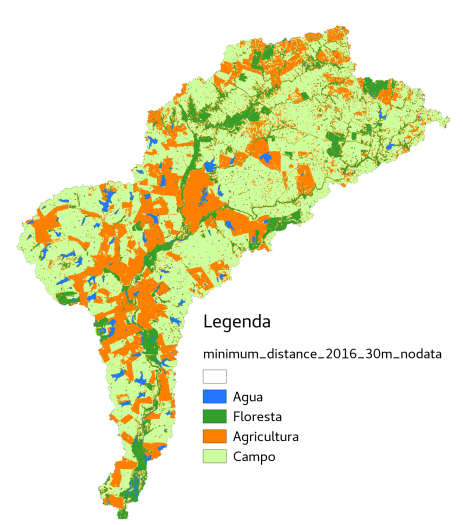

(a) 2016

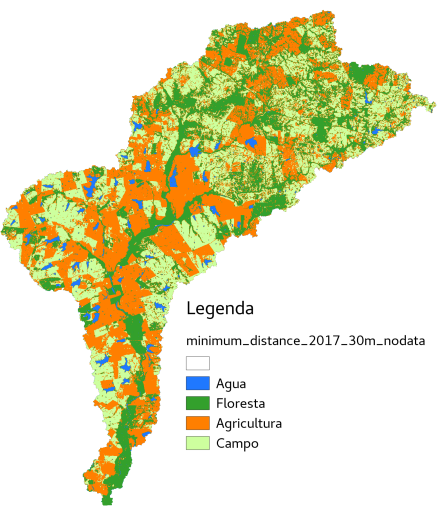

(b) 2017

\begin{tabular}{|c|c|c|c|c|c|c|c|}
\hline & Class color & 2016 & 2017 & $\Delta$ & $2016 \%$ & $2017 \%$ & $\Delta \%$ \\
\hline Agua & & 26977500.00 sq. metre & 26706600.00 sq. metre & -270900.00 sq. metre & 2.91870091276 & 2.88939218966 & -0.0293087230939 \\
\hline Floresta & & 111590100.00 sq. metre & 238747500.00 sq. metre & 127157400.00 sq. metre & 12.0729543777 & 25.830137936 & 13.7571835583 \\
\hline Agricultura & & 289738800.00 sq. metre & 382313700.00 sq. metre & 92574900.00 sq. metre & 31.3468964886 & 41.3625927217 & 10.0156962331 \\
\hline Campo & & 495991800.00 sq. metre & 276530400.00 sq. metre & -219461400.00 sq. metre & 53.6614482209 & 29.9178771526 & -23.7435710683 \\
\hline
\end{tabular}

(c) Estatísticas das Classes

Figura 4. Uso, cobertura da terra e estatísticas das classes

\begin{tabular}{|l|c|c|c|c|}
\hline & Agua & Floresta & Agricultura & Campo \\
\hline Agua & 0.839733 & 0.103386 & 0.054579 & 0.002302 \\
\hline Floresta & 0.018768 & 0.907605 & 0.058852 & 0.014776 \\
\hline Agricultura & 0.006138 & 0.163267 & 0.765084 & 0.065511 \\
\hline Campo & 0.000363 & 0.176160 & 0.307665 & 0.515812 \\
\hline
\end{tabular}

(a) Matriz de Transição 2016-2017

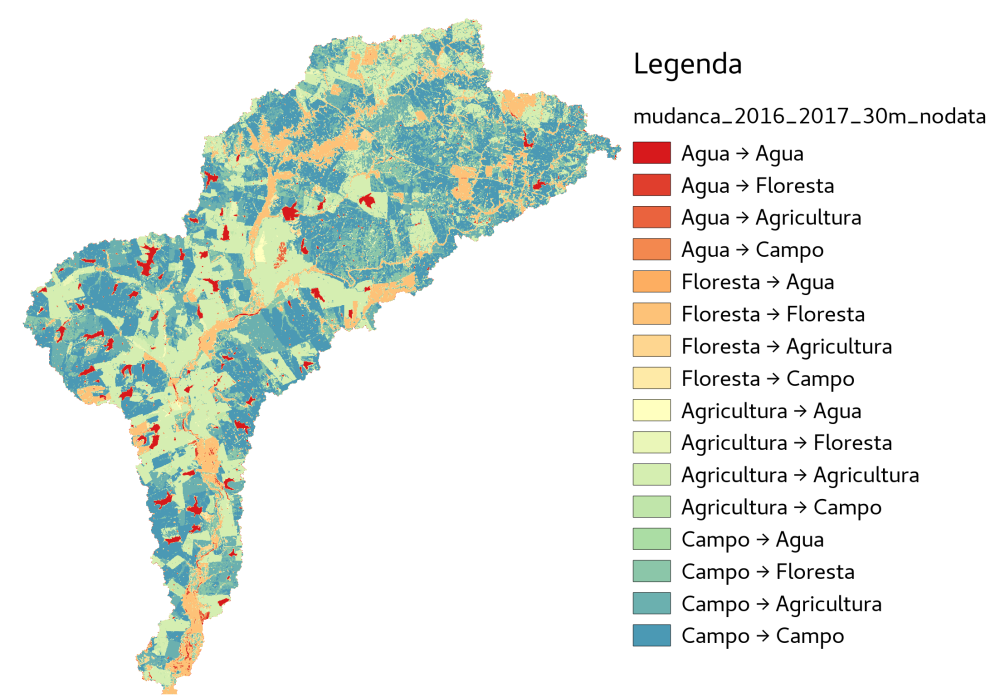

(b) Mapa de Mudanças 2016-2017

Figura 5. Matriz de transição e mapa de mudanças 2016-2017

A inferência do potencial de transição foi feito por meio de uma rede neural artificial multi-layer perceptron, onde o algoritmo de aprendizagem analisa a precisão alcançada nos conjuntos de amostras de treinamento e validação e armazena a melhor rede neural na memória. Os parâmetros utilizados nesta etapa podem ser vistos na Fi- 
gura 6. O software permite a definição do número de amostras (15) e o tipo de amostragem (estratificada), além de salvar um arquivo vetorial no formato .shp (shape file) com a localização das amostras, mas a função gerou um shape file vazio. A configuração da rede neural permite o uso de parâmetros fixos. A vizinhança define a quantidade de pixels vizinhos ao pixel corrente. O tamanho igual a 1, utilizado neste trabalho, significa 9 pixels (matriz 3x3), o tamanho igual a 2 significa 25 pixels (matriz 5x5) e assim por diante. Os parâmetros da aprendizagem foram definidos, com a taxa de aprendizagem de 0,001 , momentum em 0,001 e o número máximo de iterações em 100. Uma taxa grande de aprendizagem permite uma aprendizagem rápida, mas instável (picos no gráfico), já uma taxa de aprendizagem pequena e dinâmica significa uma aprendizagem estável, porém lenta. A quantidade de camadas ocultas é definida por uma lista de números $n_{1}, n_{2}, \ldots, n_{k}$, onde $n_{1}$ é o número de neurônios na primeira camada oculta, $n_{2}$ na segunda e assim por diante até $n_{k}$ representando o número de neurônios na última das $k$ camadas ocultas.

Os resultados da aprendizagem são apresentados na área gráfica que contém os conjuntos de erros de treinamento e de validação. O campo validação global do erro mínimo contém informação sobre o erro mínimo encontrado na validação do conjunto de amostras e o valor ficou em 0.05563 . O campo variação da precisão global mostra a diferença entre o erro encontrado e o erro atual, que ficou em 0.00057 . O campo validação kappa atual deveria mostrar o valor kappa, mas gerou um nan (not a number). Todos os parâmetros utilizados foram em função de otimizar o tempo de processamento.

No final da etapa de simulação um mapa de potencial de transição deveria mostrar a probabilidade ou potencial de mudança de uma para outra classe de uso/cobertura do solo. Os valores do potencial de transição variam de 0 (baixo potencial de transição) a 100 (alto potencial de transição). Estes mapas de potencial de transição são produzidos a partir das correspondentes mudanças de uso/cobertura do solo (por exemplo, floresta para campo ou floresta para agricultura). A função de certeza (certainty function), está em fase experimental. O resultado da simulação produz um mapa simulado do uso/cobertura da terra. Os três produtos finais (probabilidade de mudança, certeza e resultado da simulação), não foram gerados de forma adequada pelo software. A probabilidade de mudança e o mapa de certeza foram gerados sem dados e o mapa final da simulação apresenta resultado idêntico ao mapa de entrada inicial do ano de 2017. A partir desse resultado, nem foi executada a última etapa de validação, pois não havia sentido em comparar dois mapas iguais.

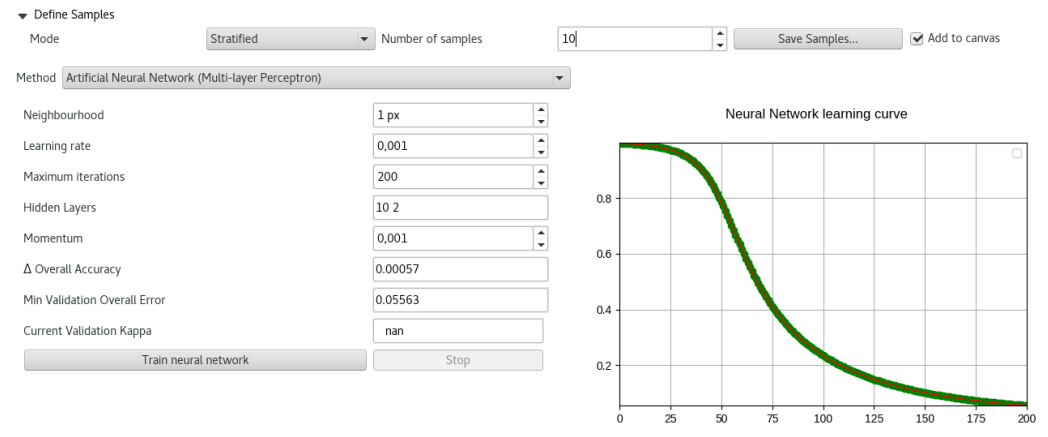

Figura 6. Modelagem Potencial de Transição 


\section{Resultados, Discussão e Conclusões}

A simulação da dinâmica da cobertura e uso do solo (ou land use and cover change LUCC) é uma área em estágio de pesquisa e desenvolvimento, de forma que as ferramentas computacionais disponíveis, principalmente as que não tem custo, apresentam diversos tipos de problemas relacionados a conceitos, implementação, documentação e/ou usabilidade. Neste trabalho foram testadas três ferramentas e cada uma delas apresentou um referencial teórico diferente com o intuito de resolver o mesmo tipo de problema. O resultado dos testes apontou estágios diferentes de desenvolvimento, referencial conceitual, estratégias de implementação, formas de documentação e correção do programa.

O TerraME é, conceitualmente, o software mais interessante e completo, pois permite que o pesquisador construa seus tipos de dados e estabeleça seu modelo, utilizando autômatos celulares ou agentes. Apresenta muitas dificuldades de instalação na plataforma Linux e documentação técnica de instalação sem muitas informações, ao ponto de não ser possível utilizar o software.

O SIMLANDER é um conjunto de comandos (script) para ser executado no software estatístico R, não tem interface gráfica e os parâmetros devem ser alterados diretamente no script. A documentação consiste em um manual de instalação e algumas apresentações feitas pela equipe e o modelo de simulação é bastante simples. Foi possível executar uma simulação com os dados de exemplo disponibilizados pelos autores, mas é necessário um pouco mais de tempo para entender detalhadamente todo o código.

O MOLUSCE consiste em um software interessante para a simulação, mas passa por alguns problemas, pois algumas funções não operaram da forma esperada e o software não informa os problemas, sendo necessário um debug avançado diretamente no código do programa para revelar os erros. Em relação ao desempenho, ele executa todas as etapas sequencialmente e em memória, ou seja, se houver um problema numa das etapas todo o trabalho é perdido e a simulação deve iniciar novamente. Para os testes, foi necessário reduzir a resolução dos arquivos de entrada de 10 metros para 30 metros, diminuindo assim o número de linhas e colunas dos arquivos matriciais.

Softwares de simulação com frequência são orientados à aplicação, visto que modelos de sistemas reais podem ser muito diversos entre si. No decorrer deste trabalho, que tem como objetivo geral estudar os processos de mudança na paisagem da região da Campanha gaúcha, evidenciou-se a necessidade de trabalhos relacionados ao desenvolvimento de plataformas robustas e corretas para simulação desses processos.

\section{Referências}

Bellomo, N. (2008). Modeling Complex Living Systems. Modeling and Simulation in Science, Engineering and Technology. Springer.

Boulic, R. and Renault, O. (1991). 3d hierarchies for animation. In Magnenat-Thalmann, N. and Thalmann, D., editors, New Trends in Animation and Visualization. John Wiley $\&$ Sons ltd.

Burrough, P. A. (1986). Principles of Geographical Information Systems for Land Resources Assessment. Monographs on Soil and Resources Survey. Clarendon Press.

Camacho, C. and Pérez-Barahona, A. (2015). Land use dynamics and the environment. Journal of Economic Dynamics and Control, 52:96-118. 
Christofoletti, A. (1999). Modelagem de Sistemas Ambientais. Edgar Blücher.

Congedo, L. (2018). Semi-automatic classification plugin documentation.

COPERNICUS (2018). Copernicus.

Câmara, G., Modesto, R. C., Souza, R., Pedrosa, B. M., Vinhas, L., Vieira, A. M., , de Carvalho Paiva, A., Tilio, M., , and Gattass, M. (2001). Terralib: Technology in support of gis innovation. page 8 .

de Senna Carneiro, T. G. (2006). Nested-CA: A foundation for multiscale modelling of land use and land cover change. PhD thesis, National Institute for Space Research (INPE), São Jos Ã@ dos Campos, Brasil.

EEA (2018). European enviroment agency.

Farr, T. G., Rosen, P. A., Caro, E., Crippen, R., Duren, R., Hensley, S., Kobrick, M., Paller, M., Rodriguez, E., Roth, L., Seal, D., Shaffer, S., Shimada, J., Umland, J., Werner, M., Oskin, M., Burbank, D., and Alsdorf, D. (2007). The shuttle radar topography mission. Reviews of Geophysics, 45(2):n/a-n/a.

Foley, J., DeFries, R., Asner, G., Barford, C., Bonan, G., Carpenter, S., Chapin, F., Coe, M., Daily, G., Gibbs, H., Helkowski, J., Holloway, T., Howard, E., Kucharik, C., Monfreda, C., Patz, J., Prentice, I., Ramankutty, N., and Snyder, P. (2005). Global consequences of land use. Science, (309):570-572.

Gomarasca, M. A. (2009). Basics of Geomatics. Springer, New York.

Ierusalimschy, R., de Figueiredo, L. H., and Filho, W. C. (1996). Lua - an extensible extension language. Software: Practice and Experience, 26(6):635-652.

Law, A. M. (2007). Simulation Modeling \& Analysis. McGraw-Hill, 4 edition.

MOLUSCE (2018). Modules for land use change evaluation.

NASA (2018). Landsat 7 science data user's handbook.

Neteler, M. and Mitasova, H. (2008). Open Source GIS: a GRASS GIS approach. Springer, New York.

OSGEO (2018). Osgeo.org your source compass.

QGIS (2018). Qgis geographic information system.

R Core Team (2018). R: A Language and Environment for Statistical Computing. $\mathrm{R}$ Foundation for Statistical Computing, Vienna, Austria.

Richards, J. A. and Jia, X. (2006). Remote Sensing Digital Image Analysis. SpringerVerlag Berlin Heidelberg, Berlin, Germany, 4 edition.

SEMA (2018). Bacia hidrográfica do rio negro.

Sentinel (2018). Sentinel online - esa.

SIMLANDER (2018). A cellular automata land use model for the $r$ software environment.

TerraME (2018). Terrame: Multiparadigm modeling toolkit. 